\title{
Nutritional Assessment
}

National Cancer Institute

\section{Source}

National Cancer Institute. Nutritional Assessment. NCI Thesaurus. Code C15286.

An in-depth evaluation of an individual's food and nutrient intake, lifestyle, and medical history designed to help the individual either maintain their health status or attain a healthier status. 